\title{
Part 7
}

\section{Pulsars and \\ other Compact Galactic Objects}




\title{
Pulsars: An Observational Overview
}

\author{
R. N. Manchester \\ Australia Telescope National Facility, CSIRO, PO Box 76, \\ Epping NSW 1710, Australia
}

\begin{abstract}
Pulsars are among the most exciting and interesting objects in the Universe. They typically have very steep radio spectra, so they are most easily observed at relatively low radio frequencies. In this review, I summarise some recent important observational results and describe recent searches for pulsars, including the very successful Parkes multibeam survey.
\end{abstract}

\section{Pulsar Spectra}

The first pulsars were discovered at a low radio frequency, $81.5 \mathrm{MHz}$, (Hewish et. 1968) and all but a handful of the 1200 presently known pulsars have been discovered at frequecies of $1.4 \mathrm{GHz}$ or less. Pulsars have steep radio spectra with a typical spectral index $(\alpha)$ of -1.7 . The first millisecond pulsar discovered, PSR $\mathrm{B} 1937+21$, has an even steeper radio spectrum, with $\alpha \sim-2.5$ (Erickson \& Mahoney 1985). This, and the fact that the next few millisecond pulsars discovered also had very steep radio spectra, led to the general belief that millisecond pulsars have steeper spectra than 'normal' pulsars. Using the sample of 281 normal pulsars and 17 millisecond pulsars detected in the Parkes $70 \mathrm{~cm}$ survey (Lyne et al. 1998), Toscano et al. (1998) showed that the mean spectral index for millisecond pulsars was about -1.9 , only marginally steeper than for normal pulsars. For both classes of pulsars the spectral indices cover a wide range, from less than -3 to $\sim 0.0$ for a few young pulsars.

Most pulsar spectra are not well described by a single power law over the whole range of observed frequencies. A compilation of spectral data for 336 pulsars by Malofeev (1999) shows that most pulsars have a spectral break at a frequency of around $1 \mathrm{GHz}$ and that many have a low-frequency turnover at $\sim 100 \mathrm{MHz}$ or below.

\section{Proper Motion and Parallax Measurements}

Accurate pulsar positions allow identification of the pulsar or its binary companion at, for example, optical or X-ray wavelengths. Measurements of changes in position, i.e. proper motion, of pulsars give information on pulsar velocities and their origin, and also on the distribution of pulsars, both alive and dead, in the Galaxy. In a few cases, position measurements are sufficiently accurate to detect the annual parallax, thereby giving model independent distances. This 
provides a basis for the pulsar distance scale and allows investigations of the distribution of interstellar electrons in the solar neighbourhood.

There are two methods by which accurate pulsar positions can be measured. The first is based on pulse timing. Fitting of the annual variations in apparent pulse period resulting from the Earth's motion around the Sun gives the pulsar position in ecliptic coordinates. The precision is of order $\Delta t / r_{E}$ radians, where $\Delta t$ is the uncertainty in pulse arrival times and $r_{E}$ is the radius of the Earth's orbit in light-seconds, about $500 \mathrm{~s}$. Since, for some millisecond pulsars at least, $\Delta t \lesssim 1 \mu \mathrm{s}$, and the precision is 0.4 mas or better.

The second method of determining accurate positions is using long-baseline interferometry, where the position is derived from the diurnal modulation of interferometric phase and hence is in celestial coordinates. These measurements have a precision is of order $\Delta \phi \lambda / B$, where $\Delta \phi$ is the uncertainty in interferometric phase, $\lambda$ is the radio wavelength and $B$ is the interferometric baseline. For VLBI, $B \sim R_{E}$, the radius of the Earth. Typically, $\Delta \phi \lambda$ is $\lesssim 1 \mathrm{~cm}$, so the position uncertainty is $\lesssim 0.3$ mas, essentially identical to that obtained from pulse timing.

Proper motions have been measured for a large sample of normal pulsars, mainly using connected interferometers (e.g. Lyne \& Lorimer 1994), showing that they typically have large space velocities, with a mean transverse velocity $\langle v\rangle \sim 350 \mathrm{~km} \mathrm{~s}^{-1}$. Proper motion measurements for millisecond pulsars, mostly obtained from pulse timing observations, show that they have smaller velocities, with $\langle v\rangle \sim 85 \mathrm{~km} \mathrm{~s}^{-1}$ (Toscano et al. 1999a). It is likely that millisecond pulsars are born as members of a binary system with a companion of several solar masses (Bhattacharya \& van den Heuvel 1991). The kick imparted by the supernova explosion then has to accelerate a system several times as massive as a single neutron star, so the resulting velocity is correspondingly less.

Postion uncertainties of $<1$ mas implies that annual parallax can be measured for pulsars at distances of $\lesssim 1 \mathrm{kpc}$. This is indeed the case and parallaxes are now known for 12 pulsars. Fig. 1a shows timing data for PSR J1744-1134 (Toscano et al. 1999b) which give a parallax for this pulsar of $2.8 \pm 0.3$ mas, corresponding to a distance of about $360 \mathrm{pc}$. The distance to this pulsar derived from the dispersion measure (DM) is less than half of this value, implying that the mean density of ionised interstellar gas along the path to this pulsar is less than the average value in the local region of the Galaxy. Fig. 1b shows measurements of position residuals from a fit of position and proper motion to data from the Very Long Baseline Array (VLBA) for PSR B0919+06 (Fomalont et al. 1999). This pulsar has a mean flux density of about $8 \mathrm{mJy}$ in the $20-\mathrm{cm}$ band and has a source suitable for phase calibration close enough to the pulsar so that both can be within the primary beam of the VLBA antennas. This greatly increases the precision and ease of phase calibration, making possible very precise astrometric measurements. The parallax derived from these measurements is $0.31 \pm 0.14$ mas. This corresonds to a distance of about $3 \mathrm{kpc}$, in good agreement with the dispersion-derived distance. 

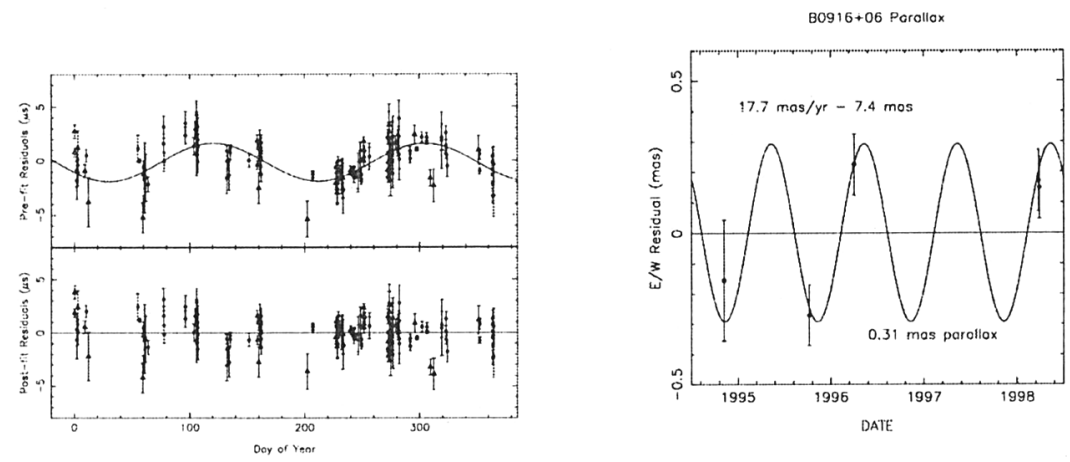

Figure 1. (a) Timing residuals for PSR J1744-1134 from data obtained at the Parkes 64-m telescope by Toscano et al. (1999b). In the upper panel, residuals from a fit of position, proper motion and pulsar spin parameters are shown; the 6-month sinusoid is the expected timing signature of an annual parallax of 2.8 mas. The lower panel shows residuals after including the parallax term in the fit. (b) Position residuals from a fit of position and proper motion to VLBA interferometric data (Fomalont et al. 1999). The observed residuals are consistent with an annual parallax of 0.31 mas.

\section{Pulse Emission Properties}

Most pulsar observations, particularly those of fluctuation and polarisation properties, give information that is relevant to our understanding of the pulse emission mechanism. In this section I briefly describe three recent reports which are of interest in this regard.

The pulsar PSR J2144-3933 was discovered in the Parkes 70-cm survey (Manchester et al. 1996) and was believed to have a period of about $2.8 \mathrm{~s}$. As part of his thesis project, Matthew Young was investigating the fluctuation properties of pulsars discovered in this survey, and realised that the pulse emission was only present every third period! The obvious conclusion was that the published period was one third of the correct value, i.e., the actual pulsar period is $8.51 \mathrm{~s}$. This is by far the longest radio pulsar period known (Young, Manchester \& Johnston 1999). It places the pulsar in a region of the $P-\dot{P}$ diagram which is beyond most published 'death lines', that is, beyond the point where pair-production can occur in the polar-cap region, given reasonable assumptions about the field line curvature.

The revised period for this pulsar results in it having an extraordinarily narrow pulse; the half-power pulse width is only $0 .^{\circ} 84$ of longitude at $1.4 \mathrm{GHz}$. This very narrow width is consistent with the emission being generated relatively close to the neutron-star surface in the core region of the polar cap. If the emitted beam is circular, it sweeps over a very small fraction of the celestial sphere. This, together with the relatively low luminosity of the pulsar, implies that the 
Galactic population of old long-period pulsars could be very large, maybe even comparable to that of 'normal' pulsars.

The phenomenon of drifting subpulses has long intrigued both observers and theoreticians. Some pulsars show a remarkable pulse modulation in which subpulses drift more-or-less uniformly across the mean profile in successive periods, usually in a backwards direction. PSR B0943+10 has a particularly complex modulation in which a backwards drift is superimposed on an odd-even fluctuation in pulse intensity. Deshpande \& Rankin (1999) have analysed a $430 \mathrm{MHz}$ pulse sequence for this pulsar recorded at Arecibo. The fluctuation spectrum shows a strong feature at 0.46 cycles per period due to the odd-even modulation. Interestingly, this feature shows symmetric sideband features with a frequency difference of 0.027 cycles per period, indicating an amplitude modulation of the phase modulation feature. This amplitude modulation is interpreted as resulting from the circulation of a ring of 20 subpulses around the periphery of the polar cap. This ingenious analysis gives an attractive image of circulating sparks, but questions remain about the uniqueness and generality of the interpretation.

Observations of mean pulse polarisation have been extremely useful in furthering our understanding of the pulse emission mechanism. Extending these observations to millisecond pulsars has been difficult because of their generally low mean flux density and the high time resolution required. An exception to this is PSR J0437-4715; this pulsar, which has a period of $5.75 \mathrm{~ms}$, has a mean flux density at $400 \mathrm{MHz}$ of nearly a Jansky and, with a DM of only $2.6 \mathrm{~cm}^{-3} \mathrm{pc}$, dispersion smearing is not a major problem. Fig. 2a shows the polarisation profiles for this pulsar at $438 \mathrm{MHz}$, obtained using the Caltech correlator system at Parkes (Navarro et al. 1997). The high signal/noise ratio makes clear the extremely complex pulse shape and polarisation variations across the wide pulse. For more highly dispersed pulsars and/or lower observing frequencies, the only feasible way to obtain high time-resolution profiles is by means of baseband recording systems. Fig. $2 \mathrm{~b}$ shows polarisation profiles for the 16-ms pulsar PSR J1022+1001, obtained at Jodrell Bank using the Princeton baseband recording system (Stairs, Thorsett \& Camilo 1999). Clearly, the polarisation variations observed in these millisecond pulsars do not fit the simple rotating-vector model of Radhakrishnan \& Cooke (1969). This suggests that the polarisation is imposed well out in the pulsar magnetosphere where the magnetic field structure is distorted by interactions occurring in the vicinity of the light cylinder.

\section{Recent Pulsar Searches}

The recent commissioning at Parkes of a 13-beam receiver operating in the 20-cm band has provided a superb instrument for searching for (and finding!) pulsars. The original motivation for development this receiver was for HI surveys (Staveley-Smith et al. 1996) but its potential for pulsar detection was realised by the construction, largely at Jodrell Bank Observatory, of a large filter-bank and data acquisition system. This system has been used for several pulsar searches since its completion in mid-1997. We briefly describe three of these searches in this section. 

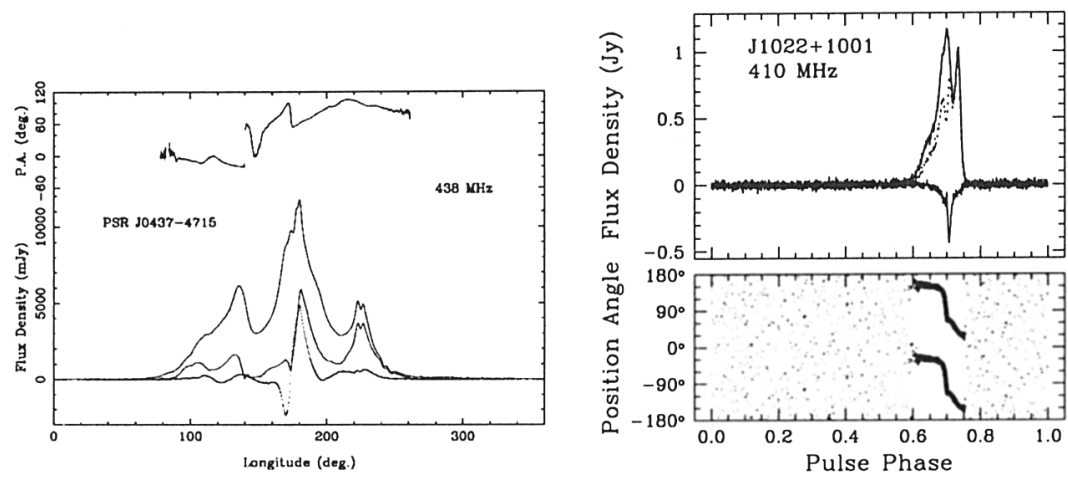

Figure 2. (a) Polarisation profiles at $438 \mathrm{MHz}$ for PSR J0437-4715 (Navarro et al. 1997). In the lower part of the plot, the upper full line is the total intensity, the lower full line is the linearly polarised intensity, and the dotted line is the circularly polarised intensity. The position angle of the linearly polarised component is shown in the upper part. (b) Polarisation profiles at $410 \mathrm{MHz}$ for PSR J1022+1001 (Stairs et al. 1999). In the upper plot the linearly polarised intensity is shown by the dotted line, and the position angle is plotted (twice) in the lower part of the figure.

\subsection{Tucanae}

The massive and relatively nearby globular cluster 47 Tucanae is well known as a rich storehouse of millisecond pulsars. All of the 11 pulsars known in the cluster prior to the present search (Robinson et al. 1995) were found using observations at $50-\mathrm{cm}$ wavelength. Recent observations with the centre beam of the multibeam receiver have been surprisingly successful in detecting not only these previously known pulsars but also others previously unknown. Nine more millisecond pulsars have been detected, all members of binary systems (Camilo et al. 1999), bringing the total number of pulsars known in the cluster to 20 , nearly a third of all known millisecond pulsars! All of these newly discovered pulsars have pulse periods between 2 and $8 \mathrm{~ms}$, as do all of the previously known ones. One of them, 47 Tuc R, has the shortest known orbital period, 1.72 hours, for any radio pulsar. Coherent timing solutions have now been obtained for most of pulsars in the cluster, allowing studies of the cluster gravitational potential and proper motion.

\subsection{Swinburne Intermediate Latitude Survey}

The Swinburne University of Technology group has undertaken a survey using the multibeam receiver at Parkes (Edwards 2000), which complements the Parkes Multibeam Pulsar Survey described below. It covers the Galactic longitude range $260^{\circ}<l<50^{\circ}$ and two strips above and below the Galactic Plane with $5^{\circ}<|b|<15^{\circ}$. The observation time per pointing is $4 \mathrm{~min}$ and the sampling interval is $125 \mu \mathrm{s}$. This survey is essentially complete and has been very 
successful, discovering 58 pulsars, eight of which have millisecond periods. Of these millisecond pulsars, six are binary with orbital periods in the range 0.4 to 22 days.

\subsection{Parkes Multibeam Pulsar Survey}

The Parkes Multibeam Pulsar Survey is a major international collaboration involving astronomers from Jodrell Bank Observatory, MIT, the University of Bologna and the ATNF (Lyne et al. 2000). It covers a strip along the Galactic Plane with $260^{\circ}<l<50^{\circ}$ and $|b|<5^{\circ}$. The central frequency is $1374 \mathrm{MHz}$ and the filterbank system provides $96 \times 3 \mathrm{MHz}$ per polarisation per beam. The observation time per pointing is 35 min, giving a limiting mean flux density at long periods of about $0.15 \mathrm{mJy}$, seven times as sensitive as the previous best survey of this type. The survey is currently about $60 \%$ complete and has been remarkably successful, with 514 confirmed new discoveries so far. When the survey commenced, in August 1997, there were about 750 pulsars known - we estimate that the complete survey will come close to doubling this number.

Timing observations to determine accurate positions, periods, period derivatives and to detect possible binary motion are made using the centre beam of the multibeam receiver for pulsars south of $-35^{\circ}$ declination, and at Jodrell Bank Observatory for pulsars north of this limit. Once a complete timing solution is obtained, which usually takes $15-18$ months from confirmation, the details are placed on the Parkes Multibeam Pulsar Survey Data Release page (http://www.atnf.csiro.au/research/pulsar/pmsurv/pmwww/release.html).

Fig. 3 is a $P-\dot{P}$ diagram showing both Parkes multibeam pulsars, previously known pulsars in the Galactic disk, and the so-called anomalous X-ray pulsars (AXPs). Three of the new pulsars have indicated surface dipole field strengths greater than any other radio pulsar (cf. Camilo et al. 2000). One of these, PSR J1814-1744, has a pulse period of $3.976 \mathrm{~s}$, which places it close to the region on the $P-\dot{P}$ diagram occupied by the AXPs. No radio emission has been detected from AXPs and, conversely, no X-ray emission has been detected from PSR J1814-1744, leaving open the question of why some of these objects are AXPs and some are radio pulsars.

Eight of the Parkes multibeam pulsars are members of binary systems. One of them is almost certainly a double-neutron-star system (Lyne et al. 2000), and five have nearly circular orbits and relatively low-mass companions which are probably white dwarfs. The other two systems, which have eccentric orbits and massive companions, are especially interesting.

For PSR J1141-6545, the orbital period is only $4.8 \mathrm{~h}$ and the orbit has an eccetricity of 0.172 (Kaspi et al. 2000). Relativistic precession of the angle of periastron has already been measured for this system and implies a total mass for the system of $2.3 \mathrm{M}_{\odot}$. At first glance, this system appears to have the characteristics of a double neutron-star system. However the pulsar is relatively young, with a characteristic age of about 1.5 Myr. This suggests that it is the second-born compact object in the system, and that the companion is a massive white dwarf, similar to that in the PSR B2303+46 system (van Kerkwijk \& Kulkarni 1999, Tauris \& Sennels 2000).

The other system is that of PSR J1740-3052 (Stairs et al. 2000); this pulsar has an orbital period of 230 days, orbit eccentricity of 0.58 , and a minimum 


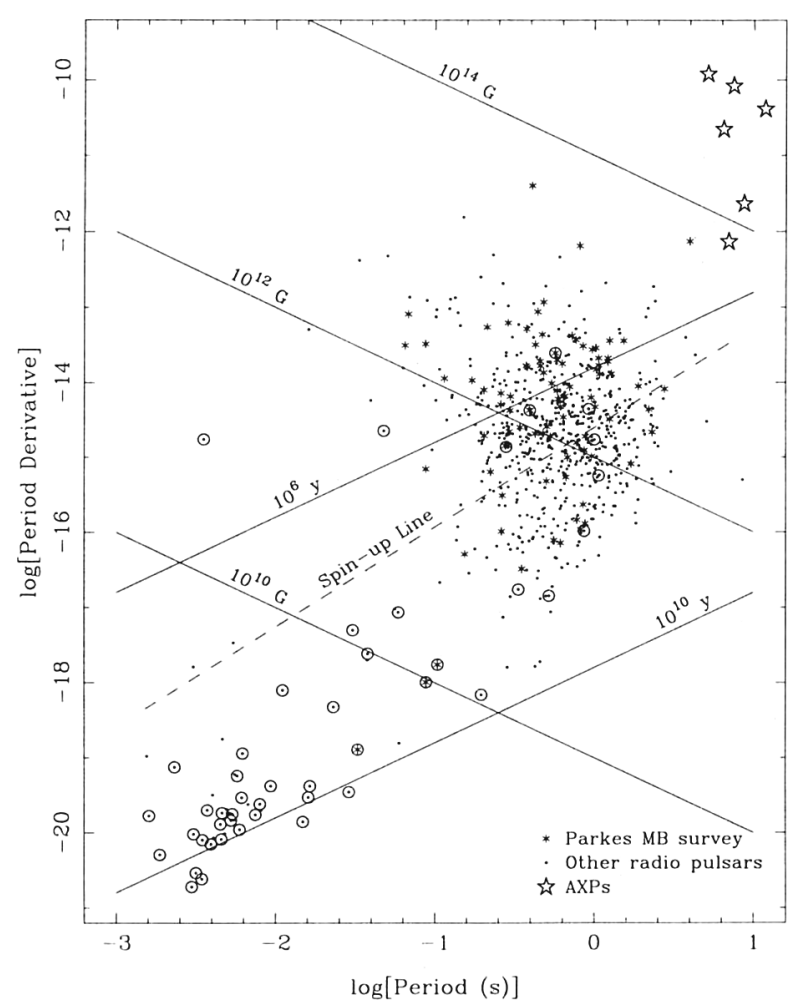

Figure 3. Period derivative versus period for the Parkes multibeam pulsars, previously known pulsars in the Galactic disk, and the anomalous X-ray pulsars (AXPs). Also shown on the figure are lines of constant characteristic age, constant surface dipole magnetic field and the spin-up line for accretion in a binary system.

companion mass of $11 \mathrm{M}_{\odot}$ ! The only possible companions for this pulsar are a normal (non-degenerate) star or a black hole. Unfortunately, the pulsar is only $2^{\circ}$ from the Galactic Centre and probably at about the same distance, so the optical field is heavily obscured. Observations in the infrared $\mathrm{K}$ band at the Anglo-Australian Telescope and the Siding Spring $2.3 \mathrm{~m}$ telescope reveal a K supergiant which is coincident with the pulsar to better than 0.3 arcsec and at a comparable distance. This star is very likely to be the companion. However, no eclipses of the pulsar emission were observed during the one periastron passage observed so far. Given that the separation of the pulsar and the companion at periastron is only 1.25 times the best estimate of the companion's radius, the lack of eclipses is puzzling. Investigations are continuing.

\section{Conclusions}

Pulsars are fascinating astrophysical objects whose study has led to valuable insight into many fields of physics and astrophysics including relativistic plasma 
physics, stellar and binary evolution, general relativity and gravitational physics, and studies of the interstellar medium. They are steep-spectrum radio sources, most easily studied at low radio frequencies. However, unlike most other studies at such frequencies, essentially all pulsar observations are sensitivity limited there is no source confusion!

The Giant Metre Wave Telescope, with its large collecting area and multiband capability, has great potential for pulsar research. As presentations at this Symposium have shown, this potential is already beginning to be realised.

\section{References}

Bhattacharya D., van den Heuvel E. P. J. 1991, Phys. Rep., 203, 1

Camilo F., Lorimer D. R., Freire P., Lyne A. G., Manchester R. N. 1999, ApJ, in press

Camilo F. et al. 2000 ApJ, submitted

Deshpande A. A., Rankin J. M. 1999, ApJ, 524, 1008

Edwards R. T. 2000, in Kramer M., Wex N., Wielebinski R., eds, Pulsar Astronomy - 2000 and Beyond, IAU Coll. 177, PASP, San Francisco, p. 33

Erickson W., Mahoney M. 1985, ApJ, 299, L29

Fomalont E. B., Goss W. M., Beasley A. J., Chatterjee S. 1999, AJ, 117, 3025

Hewish A., Bell S. J., Pilkington J. D. H., Scott P. F., Collins R. A. 1968, Nat, 217,709

Kaspi V. M. et al. 2000, ApJ, submitted

Lyne A. G., Lorimer D. R. 1994, Nat, 369, 127

Lyne A. G. et al. 1998, MNRAS, 295, 743

Lyne A. G. et al. 2000, MNRAS, in press

Malofeev V. 1999, Lebedev Physical Institute preprint

Manchester R. N. et al. 1996, MNRAS, 279, 1235

Navarro J., Manchester R. N., Sandhu J. S., Kulkarni S. R., Bailes M. 1997, ApJ, 486, 1019

Radhakrishnan V., Cooke D. J. 1969, Astrophys. Lett., 3, 225

Robinson C. R., Lyne A. G., Manchester A. G., Bailes M., D'Amico N., Johnston S. 1995, MNRAS, 274, 547

Stairs I. H. et al. 2000, ApJ, in prep.

Stairs I. H., Thorsett S. E., Camilo F. 1999, ApJS, 123, 627

Staveley-Smith L. et al. 1996, Proc. Astr. Soc. Aust., 13, 243

Toscano M., Bailes M., Manchester R., Sandhu J. 1998, ApJ, 506, 863

Toscano M., Britton M. C., Manchester R. N., Bailes M., Sandhu J. S., Kulkarni S. R., Anderson S. B. 1999a, ApJ, 523, L171

Toscano M., Sandhu J. S., Bailes M., Manchester R. N., Britton M. C., Kulkarni S. R., Anderson S. B., Stappers B. W. 1999b, MNRAS, 307, 925

van Kerkwijk M., Kulkarni S. R. 1999, ApJ, 516, L25

Young M. D., Manchester R. N., Johnston S. 1999, Nat, 400, 848 